\title{
Research on the Chinese Phonetic Teaching That Aims at the European and American Students
}

\author{
Qihan Zhu \\ Xi’an International University, Xi'an Shaanxi, 710077
}

Keywords: The European and American Students; Chinese Teaching; Phonetics Teaching; Research on Teaching

\begin{abstract}
Nowadays, as the development of globalization, the intercourses between the countries become more frequent than any time before, lots of European and American students come to China for studying. While learning of Chinese becomes the first obstacle that restricts their study abroad, and the some time, the phonetics also becomes the difficult point of their study. As the key part of Chinese teaching, the Chinese phonetic teaching becomes the disaster of the European and American students. Because these foreign students are weak in the phonetics, so when they are communicating with others, the counterparts are often confused. Especially, most of the foreign students could not differentiate the blade-alveolar and the cacuminal, and their pronunciations are often quite non-standard, so that they feel hard in their daily lives and studies. In order to change this phenomenon and improve the Chinese abilities of these European and American students, it is urgent to improve the quality of phonetic teaching and research the efficient methods of phonetic teaching, so as to improve the teaching efficient and the foreign students' Chinese abilities.
\end{abstract}

For those European and American students who come to China for studying, the most important and most elementary thing is language; this is the key factor that determines the European and American students could start their following study and life well. Only have good Chinese abilities can the students ensure their normal study and daily life smooth. As the key factor that determines whether the European and American students could learn Chinese well or not, the phonetic teaching becomes very important. At present, the conditions and situation of phonetic teaching are not optimistic, and the pronunciation of the European and American students needs to be improved much more. In order to let the European and American students learn Chinese more efficient, and to let their pronunciation more standard, it must improve the teachers' teaching abilities of phonetics and their teaching quality. The teachers should know very well about the students' characteristics and present situations on study by means of analyzing, and then find out the countermeasures, so as to improve the Chinese abilities of the students.

\section{The general statement of Chinese phonetic teaching}

For the teaching of Chinese, phonetic learning is an important part except the Pinyin and the Chinese characters; the most important thing in Chinese learning is to listen and to speak. For the European and American students, only when the students could understand the Chinese words and sentences can they understand the intentions of the speaker, and only when they could understand the meaning correctly can they begin to exchange their thinking decently by means of Chinese language [1]. The phonetic teaching could make the Chinese teaching more easily, the learners could strengthen their memories of language by means of the phonetics, and they also could strengthen their memories of the words and sentences by means of listening, these means could improve their Chinese abilities a lot. Because of the importance of the phonetics, we must pay attention to it, and highlight the status of phonetic teaching. Only in this way can we arouse the attentions of teachers and students on the phonetics. In a lot of researching results, we could easily find out that the students could have a deeper impression to a word or a sentence after they could read the word or the sentence correctly, that is the reason why the phonetic teaching is very important [2]. It is this congenital linguistic difference that the European and American students feel 
difficult to learn new languages, especially Chinese. Chinese is extensive and profound, a character may has a lot of meanings, even a character has many pronunciations, therefore, This requires the western students to be cultivated by means of the systematic phonetic training, so as to reduce the difficulty that they have in the language learning.

If the European and American students want to speak Chinese well, they must master the correct phonetics of Chinese. It is the goal of the phonetic teaching that the European and American students could speak fluent Chinese and pronounce the correct phonetics as well. It is worth to notice that this goal aims at the European and American students or other foreign students but the native people, it is not universal [3]. The circumstances where the European and American students grow up are quite different, and their language environments are also quite different, even the some words or sentences in English have the different pronunciation by the different people who are from the different areas.

\section{The difficult points of the phonetic learning for the European and American students.}

According to the investigations and researching results of the other scholars, we can divided the misunderstandings of phonetic teaching into two categories, one category is the mistakes that all the foreign students could make, the other is the mistakes that are caused by the influences of the mother tongues of the foreign students who come from different countries. The mistakes and the puzzles of the students whose mother tongues are different usually are different, but the students whose mother tongues are some often make the some mistakes on Chinese phonetic study, this shows that the mother tongue influences the study of Chinese phonetics to some extent. To solve the problem, the teachers could by means of differentiating the nationalities of the students, and divide the students into two parts; one is that whose mother tongue is the language that the words or sentences in this language have some pronunciation but different tones mean different meanings, and the other is the students whose mother tongues do not differentiate the different tones [4]. Because Chinese differentiates the different tones and the words or the sentences are the some pronunciation but different tones means different things, so the students whose mother tongues do not differentiate the different tones such as USA, Japan, South Korea etc, they may feel very difficult to learn Chinese, they have not the consciousness that the different tones means different meanings, so the image in reality is that they feel more easily on pronouncing the words than on differentiating the tones. For the students are from USA, Yu Aiqin (1986) put forward the viewpoint that the level tone is the easiest tone to learn, the falling-rising tone is the most difficult. After the research that includes the light tone, Shen Xiaonan (1989) stated that the level tone and the falling tone are more difficult than the rising tone, the falling-rising tone and the light tone [5]. Gui Mingchao (2000) pointed out that when Americans to learn Chinese Mandarin, they often are obstructed by the tone of English, this phenomenon is shown clearer when Americans read the disyllabic words, it also shows that Americans usually feel very difficult to learn Chinese Mandarin.

\section{The countermeasures for improving the Chinese phonetic learning of the European and American students.}

The phonetic studying has some difficult points, but also has some easy pronunciations to some extent, especially the Chinese language which emphasizes that the pronunciation of every word should be clear, this is difficult to the European and American students. According many relevant materials and the investigation results, we could find out that to the European and American students, the level tone is the easiest one and the falling-rising tone is the most difficult one in the four tones, i.e. the level tone (tone1), the rising tone (tone2), the falling-rising tone (tone3), and the falling tone (tone4). Most of the students can not master the changes of the different tones in Chinese [6]. Therefore, when the European and American students study Chinese, they may sort these four tones in a certain order, and begin the study from the easiest one. According to the research on the European and American students, the best order that the students could easily accept is that----tone1 first, then tone4, tone3 is after tone4, tone 2 at last. By this method that the difficulty 
degree of the phonetics is increasing step by step, the students could master the phonetic learning better, improve the accuracy of their pronunciations, and improve their Chinese abilities in the end.

To the teachers who teach Chinese phonetics to the European and American students, they ought to integrate the Chinese language with the students' mother tongues. When the students have some problems, the teachers could explain and answer the questions in English. It is very important to flexibly use Chinese and English in class, so as to improve the students' Chinese abilities. By the establishment of bilingual class, it could create a sound teaching atmosphere, and then the teachers could teach the phonetic skills and the knowledge of Chinese well in the bilingual class, at the some time, the students could learn the professional knowledge in the new teaching environment, and their Chinese phonetic abilities and Chinese cultural abilities could be improved synchronously. In the Bilingual class, the students could enrich themselves in a more realistic environment, and the integration of Chinese learning and English education becomes more perfect, it creates the good circumstances and conditions for the students' phonetic learning.

The Chinese abilities of the students could not be estimated just by the examination performances of the students, especially for the phonetic teaching, the phonetic tests and practices are more important. However, it dose not mean that we should separate the examination from the assessment, but to make the assessment more reasonable and more targeted, so that the students could improve their Chinese abilities by means of assessment. At the some time, the students should improve the accuracy and professionalism of their pronunciation and their Chinese abilities. To assess the students' overall language abilities after the test on the students' language abilities and phonetic abilities is the most important step that integrates the phonetic teaching and the Chinese learning; it also is the necessary method that improves the phonetic learning of the students. By the means of testing the student's phonetic learning situations, the students' Chinese abilities could be improved a lot.

For the European and American students, the teachers should teach Chinese to them according to their learning situations. The teachers should help the students to make their learning planning that it not only meets the students' needs of their majors, but also meet the needs of their phonetic teaching so as to help them to master Chinese quickly. In addition, the contents of the Chinese teaching should be deliberated, adopt the interest teaching methods under the premise of maintaining the professionalism, so that students can easily study from the simple knowledge to the difficult. The teachers also should let the students learn Chinese phonetics scientifically, make the specific phonetic teaching goals, analyze and solve the specific problems according to the practices during the teaching process and the teaching plans, and set up the goals that fits the development of the students, so as to improve the abilities of the students and create the good circumstances that is propitious to the phonetic learning of the students.

The professional teachers with the high qualities are the key factors that could improve the teaching level. For the phonetic teaching, the teachers are also the key factor that ensures the study effect of the students. Only the teachers that master English and Chinese could bring out high-quality professional talents [7]. And only a good Chinese teacher can combine English teaching with Chinese phonetic teaching, at the some time, takes the best of the pronunciation teaching, ensures the professionalism of teaching, and the proficiency of the students on Chinese learning. In order to let the European and American students feel easier to learn the Chinese phonetics, it is necessary to improve the phonetic teachers' language abilities and professional abilities, and take the advantages of the phonetic teaching, so as to let the students master the skills and tricks of the phonetic learning on Chinese.

\section{Conclusion}

To the European and American students, it is difficult to learn Chinese to some extent, because there are lots of differences between their native language and the Chinese language on the culture and the phonetic habit, and the complexity of the Chinese. In order to let the students, master the Chinese, it is necessary to do well in the phonetic teaching. The phonetic teaching is the key part of the Chinese teaching and the basic part as well, it determines if the European and American students 
could have the fluent oral abilities of Chinese, and it also determines if the students could build a solid foundation on Chinese learning. In order to let the European and American students have a good study conditions, the teachers must try their best to improve their teaching qualities, so as to constantly improve the students' abilities of phonetic study. Because of the author's limitation in this field, the viewpoint of the author of this thesis is not overall, so welcome all the scholars to research this issue together.

\section{References}

[1] Chen Yanwen, discuss on the Initial Consonants and the Vowels That the European and American Primary Learners Feel Difficult on Chinese [D], Nanjing University, 2015.

[2] Ding Ying, Analysis on the Phonetic Mistake of the Middle School Students in Thailand When They Study Chinese[D]. Shaanxi Normal University, 2014.

[3] Zhang Yan, Research on the Confusing Action Verbs and the Mental Verbs in the Interlanguage of the European and American Students' Chinese Learning and the Discrimination Methods[D]. Beijing Language and Culture University, 2006.

[4] Wu Menji, Gao Dingguo, Xiao Xiaoyun, Zhang Ruijian, The Acquisition Research on the Reading and Writing of Chinese for the European, American, Japan, and South Korea Students[J]. Language Teaching and Linguistic Studies, 2006.06:64-71.

[5] Song Yang, Commentary on the Acquisition Research of Learning Chinese as the Second Language[J]. Yunnan Normal University (Teaching and Research on Chinese as A Foreign Language), 2014,02:51-60.

[6] Ma Yanhua, Research on the Assimilative features on the Foreign Adults' Study of Chinese-Base on the Compared Analyze Between Chinese and other Thirteen Languages' Phonetic Systems[J]. Overseas Chinese Education, 2014,01:19-40.

[7] Tian Liang, The Factors that Influence the Phonetic Consciousness of the Foreign Students[J]. Journal of Yunnan Normal University (Teaching and Research on Chinese as A Foreign Language), 2011,01:24-28. 